\title{
Ray transference of a system with radial gradi- ent index
}

\author{
WF Harris \\ Department of Optometry, University of Johannesburg, PO Box 524, Auckland Park, 2006 South Africa
}

Received 10 October 2011; revised version accepted 28 May 2012

<wharris@uj.ac.za>

\begin{abstract}
The ray transference is central to the understanding of the first-order optical character of an optical system including the visual optical system of the eye. It can be calculated for dioptric and catadioptric systems from a knowledge of curvatures, tilts and spacing of surfaces in the system provided the material between successive surfaces has a uniform index of refraction. However the index of the natural lens of the eye is not uniform but varies with position. There is a need, therefore, for a method of calculating the transference of systems containing such gradient-index elements. As a first step this
\end{abstract}

paper shows that the transference of elements in which the index varies radially can be obtained directly from published formulae. The transferences of radial-gradient systems are examined. Expressions are derived for several properties including the power, the front- and back-surface powers and the locations of the cardinal points. Equations are obtained for rays through such systems and for the locations of images of object points through them. Numerical examples are presented in the appendix. (S Afr Optom 2012 71(2) 57-63)

Key Words: gradient index, transference, cardinal points, stigmatic system.

\section{Introduction}

Introduced into optometry under the name system matrix by Keating ${ }^{1-3}$ the ray transference, or simply the transference, is fundamental for a holistic understanding of the first-order optical character of the eye. If curvatures, tilts, separations and refractive indices are known the transference can be calculated for a dioptric system and for the visual optical system of a model eye in particular. We now also know how to calculate it for catadioptric systems including the Purkinje systems associated with the four Purkinje images in the eye ${ }^{4,5}$. The assumption underlying these analyses, however, has been that the media between successive refracting surfaces have uniform refractive indices. Applications to the eye have had to make the less-than-satisfactory-assumption that the natural lens of the eye in particular has a uniform index. An improved approach would allow one to handle materials whose indices vary with position. The question is: How can one obtain the transference of a system containing gradient-index material? Actually it is not obvious that transferences of such systems exist at all; indeed one even hears the occasional assertion that they do not exist. The purpose of this paper is to show that the transference does exist for a particular gradient-index system and to obtain an explicit expression for it. The index of the medium varies radially, that is, perpendicular to the optical axis.

Marchand ${ }^{6}$ presents equations for the paraxial behaviour of rays in a system in which the index of refraction is a parabolic function of radius. We summarize these equations and then show how they lead directly to expressions for the transferences of systems made up of such material. We check that the transferences are symplectic and examine some of their features. We illustrate the use of the transferences by obtaining expressions for several properties of the system including the power, vertex powers and the locations of the cardinal points of the systems. 


\section{Marchand's equations}

Figure 1 represents an optical system $S$ with longitudinal axis $\mathrm{Z}$ and entrance and exit planes $\mathrm{T}_{0}$ and $\mathrm{T}_{1}$. $\mathrm{Z}$ is in fact the optical axis of the system. The radius $r$ is measured from and orthogonal to $Z$. The index $n$ varies radially, that is, $n=n(r)$. Upstream of $\mathrm{T}_{0}$ the index $n_{0}$ is uniform and downstream of $\mathrm{T}_{1}$ the index $n_{1}$ is uniform.

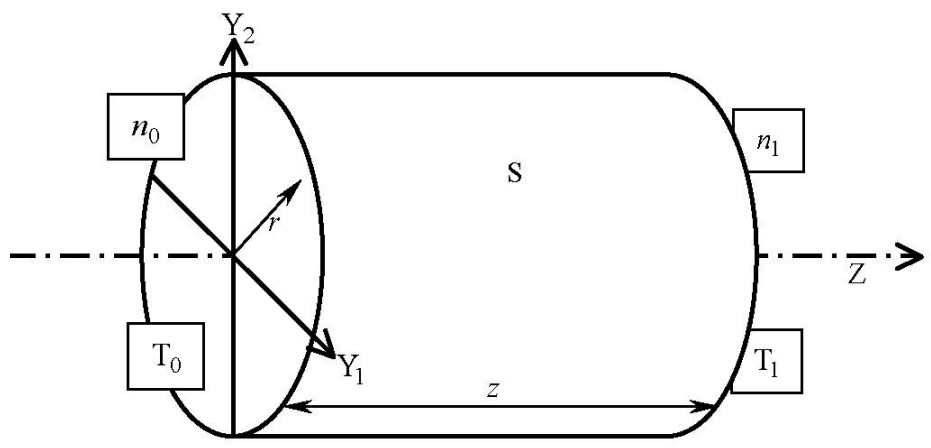

Figure 1 A radial-gradient system $\mathrm{S}$ of length $z$. $\mathrm{Z}$ is the longitudinal axis and $T_{0}$ and $T_{1}$ the entrance and exit planes respectively. Within $\mathrm{S}$ the index is a parabolic function of radius $r$. Outside $\mathrm{S}$ the index is uniform being $n_{0}$ upstream of $\mathrm{T}_{0}$ and $n_{0}$ downstream of $\mathrm{T}_{1} . \mathrm{Y}_{1}$ and $\mathrm{Y}_{2}$ are horizontal and vertical axes respectively.

Following Marchand ${ }^{6}$ we represent the index as an infinite series in $r$ and truncate after the term in $r^{2}$. In order to make the function invariant under rotation about axis $\mathrm{Z}$ we remove the term in $r$. The result is the parabolic equation

$n=n_{\mathrm{a}}+k r^{2}$

with two constants $n_{\mathrm{a}}$ and $k . n_{\mathrm{a}}$ is the axial index of refraction. For $k<0$ the index decreases away from axis Z; for $k>0$ it increases away from Z. We shall refer to $S$ in the former case as a decreasing radialgradient system and in the latter case as an increasing radial-gradient system.

Consider a ray traversing system $\mathrm{S}$. It is incident with transverse position $\mathbf{y}_{0}=\left(\begin{array}{l}y_{01} \\ y_{02}\end{array}\right)$ and emerges at transverse position $\mathbf{y}_{1}=\left(\begin{array}{l}y_{11} \\ y_{12}\end{array}\right)$. The Cartesian coordinates are with respect to horizontal and vertical axes $Y_{1}$ and $Y_{2}$ orthogonal to $Z$. The ray's inclina- tions at incidence and emergence are $\mathbf{a}_{0}$ and $\mathbf{a}_{1}$ respectively, also with coordinates with respect to horizontal and vertical axes just as for $\mathbf{y}_{0}$ and $\mathbf{y}_{1}$. The reduced inclinations are $\boldsymbol{\alpha}_{0}=n_{0} \mathbf{a}_{0}$ and $\boldsymbol{\alpha}_{1}=n_{1} \mathbf{a}_{1}$.

Snell's equation for refraction applied across the first surface of the system shows that, although the inclination changes, the reduced inclination does not. It follows that $\mathbf{y}_{0}$ and $\boldsymbol{\alpha}_{0}$, defined immediately outside the system, also represent the transverse position and reduced inclination immediately inside the system. Similarly $\mathbf{y}_{1}$ and $\boldsymbol{\alpha}_{1}$ do not change across the last surface of the system.

Marchand ${ }^{6}$ defines

$b=\sqrt{\left|2 k n_{\mathrm{a}}\right|}$

and

$\eta=b z / n_{\mathrm{a}}$.

$b$ is a reciprocal length (it has the same units as dioptric power). $\eta$ is unitless; it increases in direct proportion to the length $z$. For some purposes it is useful to think of it as an angle (in radians).

For decreasing radial-gradient systems $(k<0)$ Marchand shows that paraxial rays emerge with components of transverse position

$y_{11}=y_{01} \cos \eta+\frac{\alpha_{01}}{b} \sin \eta$

and

$y_{12}=y_{02} \cos \eta+\frac{\alpha_{02}}{b} \sin \eta$

and reduced inclination

$\alpha_{11}=\alpha_{01} \cos \eta-b y_{01} \sin \eta$

and

$\alpha_{12}=\alpha_{02} \cos \eta-b y_{02} \sin \eta$.

For increasing radial-gradient systems $(k>0)$ the equations are similar but are in terms of hyperbolic functions:

$y_{11}=y_{01} \cosh \eta+\frac{\alpha_{01}}{b} \sinh \eta$

and

$y_{12}=y_{02} \cosh \eta+\frac{\alpha_{02}}{b} \sinh \eta$

and reduced inclination

$\alpha_{11}=\alpha_{01} \cosh \eta+b y_{01} \sinh \eta$

and 
$\alpha_{12}=\alpha_{02} \cosh \eta+b y_{02} \sinh \eta$.

Note the presence of minus signs in Equations 6 and 7 and their absence in corresponding Equations 10 and 11 .

\section{Transferences}

Equations 4 to 7 can be combined into the single matrix equation

$\left(\begin{array}{cccc}\cos \eta & 0 & \frac{1}{b} \sin \eta & 0 \\ 0 & \cos \eta & 0 & \frac{1}{b} \sin \eta \\ -b \sin \eta & 0 & \cos \eta & 0 \\ 0 & -b \sin \eta & 0 & \cos \eta\end{array}\right)\left(\begin{array}{l}y_{01} \\ y_{02} \\ \alpha_{01} \\ \alpha_{02}\end{array}\right)=\left(\begin{array}{l}y_{11} \\ y_{12} \\ \alpha_{11} \\ \alpha_{12}\end{array}\right)$

as multiplication readily confirms. Hence we can write

$\left(\begin{array}{cc}\mathbf{I} \cos \eta & \mathbf{I} \frac{1}{b} \sin \eta \\ -\mathbf{I} b \sin \eta & \mathbf{I} \cos \eta\end{array}\right)\left(\begin{array}{l}\mathbf{y}_{0} \\ \boldsymbol{\alpha}_{0}\end{array}\right)=\left(\begin{array}{l}\mathbf{y}_{1} \\ \boldsymbol{\alpha}_{1}\end{array}\right)$

where $\mathbf{I}$ is the $2 \times 2$ identity matrix. Thus we have the basic equation of linear optics

$\mathbf{S} \boldsymbol{\rho}_{0}=\boldsymbol{\rho}_{1}$

where

$\mathbf{S}=\left(\begin{array}{cc}\mathbf{I} \cos \eta & \mathbf{I} \frac{1}{b} \sin \eta \\ -\mathbf{I} b \sin \eta & \mathbf{I} \cos \eta\end{array}\right)$

is the transference of the system. Equation 15, therefore, gives the $4 \times 4$ transference of a decreasing radial-gradient system with $b$ and $\eta$ defined by Equations 2 and 3 respectively.

In the same way Equations 8 to 11 lead to the conclusion that

$\mathbf{S}=\left(\begin{array}{cc}\mathbf{I} \cosh \eta & \mathbf{I} \frac{1}{b} \sinh \eta \\ \mathbf{I} b \sinh \eta & \mathbf{I} \cosh \eta\end{array}\right)$

is the transference of an increasing radial-gradient system. Note the minus sign in Equation 15 and its absence in Equation 16.
Because of the general expression

$\mathbf{S}=\left(\begin{array}{ll}\mathbf{A} & \mathbf{B} \\ \mathbf{C} & \mathbf{D}\end{array}\right)$

Equations 15 and 16 give the dilation $\mathbf{A}$, the disjugacy $\mathbf{B}$, the divergence $\mathbf{C}$ and the divarication $\mathbf{D}$ of a system with a radial gradient. Equations 15 and 16 show explicitly that such systems have transferences of the form

$\mathbf{S}=\left(\begin{array}{cc}\mathbf{I} A & \mathbf{I} B \\ \mathbf{I} C & \mathbf{I} D\end{array}\right)$

which confirms that they are stigmatic. $A, B, C$ and $D$ are the scalar dilation, scalar disjugacy, etc., of the system.

It is immediately apparent from Equations 15 and 16 that

A $=$ D

for both increasing and decreasing radial-gradient systems.

\section{Symplecticity}

Before venturing further we need to confirm that the transferences are symplectic ${ }^{7}$, that is, that they satisfy

$\mathbf{S}^{\mathrm{T}} \mathbf{E S}=\mathbf{E}$.

where

$$
\mathbf{E}:=\left(\begin{array}{cc}
\mathbf{O} & \mathbf{I} \\
-\mathbf{I} & \mathbf{O}
\end{array}\right) .
$$

Substitution from Equations 15 and 16 shows that they are indeed symplectic. Demonstrating symplecticity in the case of Equation 16 requires use of the identity

$(\cosh \eta)^{2}-(\sinh \eta)^{2}=1$

\section{Decreasing radial-gradient systems}

Consider systems with $k<0$; the index is a maximum on longitudinal axis $\mathrm{Z}$ and decreases radially outward. The transference is given by Equation 15 . A numerical example is given in the Appendix.

The scalar fundamental properties are all represented as sine waves in the length $z$ of the system. Their magnitudes are given by

$|A|=|D| \leq 1$, 
$|B| \leq 1 / b$

and

$|C| \leq b$.

The disjugacy and divergence always have opposite signs. For $z=0$ Equation 3 reduces to $\eta=0$ and, hence, Equation 15 reduces, as expected, to

$\mathbf{S}=\mathbf{I}$

where $\mathbf{I}$ is the $4 \times 4$ identity matrix. Because $b$ and $n_{\text {a }}$ are always positive $\eta$ always increases from 0 as $z$ increases from 0 . Hence the scalar dilation $A=\cos \eta=D$ decreases from 1 while the scalar disjugacy $B=\frac{1}{b} \sin \eta$ increases from 0 and the scalar divergence $C=-b \sin \eta$ decreases from 0 . When $z=\pi n_{\mathrm{a}} / 2 b$,

that is, $\eta=\pi / 2$ (by Equation 3),

$\mathbf{S}=\left(\begin{array}{cc}\mathbf{O} & \mathbf{I} \frac{1}{b} \\ -\mathbf{I} b & \mathbf{O}\end{array}\right)$.

When

$z=\pi n_{\mathrm{a}} / b$

$\eta=\pi$ and the transference of the system is

$\mathbf{S}=-\mathbf{I}$.

When

$z=z_{\mathrm{m}}=2 \pi n_{\mathrm{a}} / b$

$\eta=2 \pi$ and the transference is given by Equation 26 again. The same is true for $z$ equal to any integral multiple of $z_{\mathrm{m}}$. We see that decreasing radial-gradient systems are periodic in $z$ with period $z_{\mathrm{m}}$. One can think of $z_{\mathrm{m}}$ as the wavelength of the system.

By definition ${ }^{8}$ the dioptric power of a system is $\mathbf{F}=-\mathbf{C}$. Thus a decreasing radial-gradient system has power

$\mathbf{F}=\mathbf{I} b \sin \eta$.

Its scalar power is $F=b \sin \eta$. Depending on the length of the system its scalar power lies between $-b$ and $b$.

In general the back- and front-vertex powers of a system are given by $\mathbf{F}_{\mathrm{bv}}=\mathbf{F} \mathbf{A}^{-1}$ and $\mathbf{F}_{\mathrm{fv}}=\mathbf{D}^{-1} \mathbf{F}$ respectively. ${ }^{9}$ It follows that, for a decreasing radialgradient system,

$\mathbf{F}_{\mathrm{bv}}=\mathbf{F}_{\mathrm{fv}}=\mathbf{I} b \tan \eta$.

Relative to the entrance plane an incident special point of characteristic $X$ has axial position $z_{\mathrm{Q} 0}=n_{0}(D-X) / C$ and relative to the exit plane an emergent special point has axial position $z_{\mathrm{Q} 1}=-n_{1}(A-1 / X) / C \cdot .^{10,11}$ Thus a decreasing radial-gradient system has incident and emergent special points with locations

$z_{\mathrm{Q} 0}=-n_{0} \frac{\cos \eta-X}{b \sin \eta}$

and

$z_{\mathrm{Q} 1}=n_{1} \frac{\cos \eta-1 / X}{b \sin \eta}$

respectively. It follows that the cardinal points of the system are located as summarized in Table 1.

Table 1 Axial positions $z_{\mathrm{Q} 0}$ and $z_{\mathrm{Q} 1}$ and characteristic $X$ of the incident and emergent cardinal points $\mathrm{Q}_{0}$ and $\mathrm{Q}_{1}$ relative to the entrance $T_{0}$ and exit $T_{1}$ planes of a decreasing radial-gradient system.

\begin{tabular}{lllll}
\hline Cardinal point & Symbol & Characteristic $X$ & Axial position \\
\hline \multirow{4}{*}{ Incident } & Focal & $\mathrm{F}_{0}$ & 0 & $z_{\mathrm{F} 0}=-n_{0} /(b \tan \eta)$ \\
& Principal & $\mathrm{P}_{0}$ & 1 & $z_{\mathrm{P} 0}=-n_{0}(\cos \eta-1) /(b \sin \eta)$ \\
& Nodal & $\mathrm{N}_{0}$ & $n_{1} / n_{0}$ & $z_{\mathrm{N} 0}=\left(n_{0} \cos \eta-n_{1}\right) / b \sin \eta$ \\
& - & & $\infty$ & $\infty$ \\
\hline \multirow{4}{*}{ Emergent } & Principal & $\mathrm{P}_{1}$ & 1 & $\infty$ \\
& Nodal & $\mathrm{N}_{1}$ & $n_{1} / n_{0}$ & $z_{\mathrm{P} 1}=n_{1}(\cos \eta-1) /(b \sin \eta)$ \\
& Focal & $\mathrm{F}_{1}$ & $\infty$ & $z_{\mathrm{N} 1}=\left(n_{1} \cos \eta-n_{0}\right) /(b \sin \eta)$ \\
\hline
\end{tabular}


Let us now extend the system by adding a homogeneous gap of width $z_{0}$ and index $n_{0}$ upstream (reduced width $\zeta_{0}=z_{0} / n_{0}$ ) and a second homogeneous gap of width $z_{1}$ and index $n_{1}$ downstream. The compound system has transference

$\left(\begin{array}{cc}\mathbf{I} & \mathbf{I} \boldsymbol{\zeta}_{1} \\ \mathbf{O} & \mathbf{I}\end{array}\right)\left(\begin{array}{cc}\mathbf{I} \cos \eta & \mathbf{I} \frac{1}{b} \sin \eta \\ -\mathbf{I} b \sin \eta & \mathbf{I} \cos \eta\end{array}\right)\left(\begin{array}{cc}\mathbf{I} & \mathbf{I} \boldsymbol{\zeta}_{0} \\ \mathbf{O} & \mathbf{I}\end{array}\right)(36)$

Multiplication shows that the compound system has scalar disjugacy

$\left(\zeta_{0}+\zeta_{1}\right) \cos \eta+\left(\frac{1}{b}-b \zeta_{0} \zeta_{1}\right) \sin \eta$

Equating this expression to 0 gives the condition that the compound system is conjugate, that is, an object point on its entrance plane will produce an image point on its exit plane. Equating the expression to 0 and solving we obtain

$\zeta_{1}=\frac{\zeta_{0}+\frac{1}{b} \tan \eta}{b \zeta_{0} \tan \eta-1}$.

This equation gives the axial location of the image of an object point through the decreasing radial-gradient system.

From Equations 13 and 3 we obtain

$\mathbf{y}_{0} \cos \frac{b z}{n_{\mathrm{a}}}+\boldsymbol{\alpha}_{0} \frac{1}{b} \sin \frac{b z}{n_{\mathrm{a}}}=\mathbf{y}_{1}$.

This is simply a combination of two of Marchand's 6 equations (Equations 4 and 5). It is the equation through the system of a ray that is incident with transverse position $\mathbf{y}_{0}$ and reduced inclination $\boldsymbol{\alpha}_{0}$. In particular consider a ray that enters the system at transverse position $\left(\begin{array}{c}0 \\ y_{02}\end{array}\right)$ and with reduced inclination $\left(\begin{array}{c}\alpha_{01} \\ 0\end{array}\right)$. Equation 39 reduces to
$\left(\begin{array}{c}\alpha_{01} \frac{1}{b} \sin \frac{b z}{n_{\mathrm{a}}} \\ y_{02} \cos \frac{b z}{n_{\mathrm{a}}}\end{array}\right)=\left(\begin{array}{c}y_{11} \\ y_{12}\end{array}\right)$.
If $\alpha_{01}=0$, that is, the ray is incident parallel to longitudinal axis $\mathrm{Z}$, then the ray obeys

$$
y_{02} \cos \frac{b z}{n_{\mathrm{a}}}=y_{12} \text {. }
$$

The ray is a sine wave in the vertical meridian. If, instead, $\alpha_{01}=b y_{02}$ then it follows from Equation 40 that $y_{11}^{2}+y_{12}^{2}=y_{02}^{2}$. This means that at every cross section along the system the ray is at the same distance $r=\left|y_{02}\right|$ from axis Z. Equation 40 becomes the parametric equation of a circular helix. In fact the ray is a helix with pitch $z_{\mathrm{m}}$ (Equation 31), radius $r$ and axis $\mathrm{Z}$.

\section{Increasing radial-gradient systems}

For $k>0$ the index increases radially outward from a minimum on longitudinal axis $Z$. The transference is given by Equation 16. See the Appendix for a numerical example.

\section{Now}

$\sin \eta=\left(e^{\eta}-e^{-\eta}\right) / 2$

and

$\cosh \eta=\left(e^{\eta}+e^{-\eta}\right) / 2$.

It follows from Equation 43 that

$A=D \geq 1$

and from Equation 42 that

$B \geq 0$

and

$C \geq 0$.

Thus none of the entries of the transference of a decreasing radial-gradient system can be negative. For $z=0$ the transference is again the identity matrix (Equation 26). As $z$ increases all the scalar fundamental properties increase monotonically without limit. In strong contrast to the case with decreasing radialgradient systems there is no periodic behaviour.

From Equation 16 we see that

$\mathbf{F}=-\mathbf{I} b \sin \eta$.

This shows that $F \leq 0$. Thus the scalar power decreases from 0 without limit as the length $z$ of the system increases. The scalar power of every increasing radial-gradient system of positive thickness is negative. This contrasts with decreasing radial-gradient systems whose power may be negative, zero or positive.

The front- and back-vertex powers are given by 
$\mathbf{F}_{\mathrm{bv}}=\mathbf{F}_{\mathrm{fv}}=-\mathbf{I} b \tanh \eta$.

Thus as $z$ increases from 0 the scalar vertex powers decrease from 0 and approach the fixed value $-b$ for large $z$. $(\tanh \eta \rightarrow 1$ as $\eta \rightarrow \infty$.)

The special points are located according to

$$
z_{\mathrm{Q} 0}=n_{0} \frac{\cosh \eta-X}{b \sinh \eta}
$$

and

$$
z_{\mathrm{Q} 1}=-n_{1} \frac{\cosh \eta-1 / X}{b \sinh \eta}
$$

The locations of the cardinal points in particular are listed in Table 2.

Following the same procedure used above in the case of decreasing radial-gradient systems we find that an image point is located according to

$\zeta_{1}=-\frac{\zeta_{0}+\frac{1}{b} \tanh \eta}{b \zeta_{0} \tanh \eta+1}$

A ray through the system obeys

$$
\mathbf{y}_{0} \cosh \frac{b z}{n_{\mathrm{a}}}+\boldsymbol{\alpha}_{0} \frac{1}{b} \sinh \frac{b z}{n_{\mathrm{a}}}=\mathbf{y}_{1} .
$$

In particular for $\mathbf{y}_{0}=\left(\begin{array}{c}0 \\ y_{02}\end{array}\right)$ and $\boldsymbol{\alpha}_{0}=\left(\begin{array}{c}\alpha_{01} \\ 0\end{array}\right)$

$$
\left(\begin{array}{c}
\alpha_{01} \frac{1}{b} \sinh \frac{b z}{n_{\mathrm{a}}} \\
y_{02} \cosh \frac{b z}{n_{\mathrm{a}}}
\end{array}\right)=\left(\begin{array}{l}
y_{11} \\
y_{12}
\end{array}\right) .
$$

Both components grow without limit; the ray deviates increasingly from the longitudinal axis.

\section{Concluding remarks}

We have obtained here the transferences of optical systems with refractive index that varies radially. As far as we are aware this represents the first calculation of a transference of a system containing gradientindex material.

Radial-gradient systems fall into two classes, those with index a maximum along the optical axis and those with the index a minimum along the axis. In the case of the former the index falls off with increasing distance from the axis; such systems have transferences given by Equation 15. The trigonometric functions give the system a periodic structure. Rays in effect are attracted towards the optical axis. They travel through the system oscillating or rotating about the axis. An optical fibre is an example of a system of this type. On the other hand radial-gradient systems in which the index increases away from the axis have no periodic structure (the transference is given by Equation 16); rays tend to be repelled, as it were, by the optical axis. They splay outwards at increasing angles and tend to leave the system at its sides.

The transferences of radial-index systems are shown explicitly to be symplectic as they must be in linear optics.

Formulae have been derived here for the power and front- and back-vertex powers of radial-index systems and for the locations of their cardinal and

Table 2 Axial positions $z_{\mathrm{Q} 0}$ and $z_{\mathrm{Q} 1}$ and characteristic $X$ of the incident and emergent cardinal points $\mathrm{Q}_{0}$ and $\mathrm{Q}_{1}$ relative to the entrance $\mathrm{T}_{0}$ and exit $\mathrm{T}_{1}$ planes of a decreasing radial-gradient system.

\begin{tabular}{lllll}
\hline Cardinal point & Symbol & Characteristic $X$ & Axial position \\
\hline \multirow{4}{*}{ Incident } & Focal & $\mathrm{F}_{0}$ & 0 & $z_{\mathrm{F} 0}=n_{0} /(b \tanh \eta)$ \\
& Principal & $\mathrm{P}_{0}$ & 1 & $z_{\mathrm{P} 0}=n_{0}(\cosh \eta-1) /(b \sinh \eta)$ \\
& Nodal & $\mathrm{N}_{0}$ & $n_{1} / n_{0}$ & $z_{\mathrm{N} 0}=\left(n_{0} \cosh \eta-n_{1}\right) /(b \sinh \eta)$ \\
& - & & $\infty$ & $\infty$ \\
\hline \multirow{5}{*}{ Emergent } & & & 0 & $\infty$ \\
& Podal & $\mathrm{N}_{1}$ & $n_{1} / n_{0}$ & $z_{\mathrm{P} 1}=-n_{1}(\cosh \eta-1) /(b \sinh \eta)$ \\
& Focal & $\mathrm{F}_{1}$ & $\infty$ & $z_{\mathrm{N} 1}=-\left(n_{1} \cosh \eta-n_{0}\right) /(b \sinh \eta)$ \\
\hline
\end{tabular}


other special points. The systems form images of objects. Formulae for the locations of image points are presented.

\section{Acknowledgments}

I gratefully acknowledge support from the National Research Foundation and continuing discussions with RD van Gool, T Evans and A Rubin.

\section{Appendix}

We make the arbitrary choice of $n_{\mathrm{a}}=1.45$ and $k$ $=-0.002 \mathrm{~mm}^{-2}$ in Equation 1 and choose the system to have length $z=5 \mathrm{~mm}$. Because $k$ is negative the system is a decreasing radial-gradient system. Equations 2 and 3 give $b=0.0762 \mathrm{~mm}^{-1}$ and $\eta=0.2626$. Equation 31 shows that the system has wavelength of $z_{\mathrm{m}}=119.6 \mathrm{~mm}$. From Equation 15 we obtain the transference of the system:

$$
\mathbf{S}=\left(\begin{array}{cc}
0.9657 \mathbf{I} & 3.4088 \mathbf{I} \mathrm{mm} \\
-0.0198 \mathbf{I} \mathrm{mm}^{-1} & 0.9757 \mathbf{I}
\end{array}\right)
$$

Thus the scalar power of the system is $F=19.8 \mathrm{D}$ and the scalar vertex powers $F_{\mathrm{bv}}=F_{\mathrm{fv}}=20.5 \mathrm{D}$.

We retain the same numerical values except that now we reverse the sign of $k$, that is, $k=0.002 \mathrm{~mm}^{-2}$. Now we have an increasing radial-gradient system. $b$ and $\eta$ are unchanged. From Equation 16 we find that the system's transference is

$$
\mathbf{S}=\left(\begin{array}{cc}
1.0347 \mathbf{I} & 3.4880 \mathbf{I} \mathrm{mm} \\
0.0202 \mathbf{I} \mathrm{mm}^{-1} & 1.0347 \mathbf{I}
\end{array}\right)
$$

Its scalar power is $F=-20.2 \mathrm{D}$ and its scalar vertex powers are $F_{\mathrm{bv}}=F_{\mathrm{fv}}=-19.6 \mathrm{D}$.

\section{References}

1. Keating MP. A system matrix for astigmatic optical systems: I. Introduction and dioptric power relations. Am J Optom Physiol Opt 198158 810-819.

2. Keating MP. A system matrix for astigmatic optical systems: II. Corrected systems including an astigmatic eye. Am J Optom Physiol Opt 198158 919-929.

3. Keating MP. Advantages of a block matrix formulation for an astigmatic system. Am J Optom Physiol Opt 198259 851-857.

4. Harris WF. Transferences of heterocentric astigmatic catadioptric systems including Purkinje systems. Optom Vis Sci 201087 778-786.

5. Harris WF. Transferences of Purkinje systems. S Afr Optom 201170 57-60.
6. Marchand EW. Gradient Index Optics. Academic Press, New York 1978 67-69.

7. Harris WF. Symplecticity and relationships among the fundamental properties in linear optics. S Afr Optom 201069 3-13.

8. Harris WF. Dioptric power: its nature and its representation in three- and four-dimensional space. Optom Vis Sci 1997 74 349-366.

9. Harris WF. Back- and front-vertex powers of astigmatic systems. Optom Vis Sci 201087 70-72.

10. Harris WF. Cardinal points and generalizations. Ophthal Physiol Opt 201030 391-401.

11. Harris WF. Pascal's ring, cardinal points, and refractive compensation. Vision Res 201151 1679-1685. 